\title{
Pacing Lead/Myocardium Interface: Modelling and Characterization of the Impedance
}

\author{
A Gressard $^{1}$, C Besnard ${ }^{1}$, D Dubet ${ }^{2}$, P Lantelme ${ }^{1,3}$, H Milon ${ }^{1,3}$ \\ ${ }^{1}$ Croix Rousse Hospital, Cardiology Department, Lyon, France \\ ${ }^{2}$ Centre de Recherches Astronomiques de Lyon, Lyon, France \\ ${ }^{3}$ UMR-MA 103, University of Lyon I, Lyon, France
}

\begin{abstract}
The electrical pattern of the pacing lead/myocardium interface can be simplified as a resistor $R$ with, in parallel, a capacitor $C$ and a resistor $r$. Our work is to evaluate these three impedance components of the interface. We fed a voltage across a serial resistor in the pacing lead circuit and we measured, sampled, digitized and recorded it. Then we calculated the electrical current through the pacing lead circuitry. The applied pacing waveform signal is a square wave at constant voltage and the current can be modeled as follows: $I(t)=V\left(a \cdot e^{b t}+\right.$ c). Using a digital analysis method, we assessed $a, b$ and $c$ whence we inferred the components of the interface impedance ( $R, r$ and $C)$. The latter characterize the interface polarization and allow the calculation of the polarization voltage whatever the pacing waveform. Moreover, with this method, the characteristics of any pacing lead can easily be determined during pace-maker implantations.
\end{abstract}

\section{Introduction}

The interface between the pacing electrode and the endocardium is more than just a simple ohmic type electrical contact [1,2]. This is evident from the comparison of the old signals obtained by photoanalysis with the pacing pulse output by the pacemaker. This pulse measured across the pacemaker terminals is a voltage pulse whereas the signal measured by skin electrodes is the current image of this pulse. The morphological differences between voltage and current signals are due to the composite electrical nature of the heart-electrode interface.

\subsection{Purpose and interest of the study}

We propose to model the heart-electrode interface as an electric circuit and to determine the value of each of the components. This will make it possible to compare electrodes (of different models and within a given model), establish the role of the blood and endocardium by proceeding measurements under different conditions as well as to determine the polarization of the interface universally from knowledge of the polarization circuit and the applied signal (a voltage or current signal of known duration).

\subsection{Model, theoretical analysis}

The contact between the electrode and endocardium in the blood electrolytes electrically forms a capacitor C. This capacitor, highly imperfect, is shunted by its leakage resistor $\mathrm{R}$ connected in parallel with $\mathrm{C}$. Resistor $\mathrm{r}$, in series with this parallel $\mathrm{RC}$ circuit, corresponds to the ohmic resistance of the wire, the tissue resistance and the plasma resistance (electrolytes) [3-7].

The simplest model is therefore the electric circuit below:

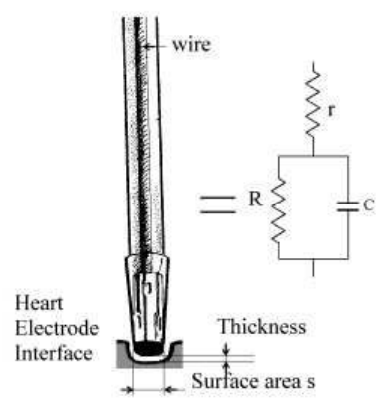

We will neglect the inductive effect due to the spiral structure of the wire, because our measurements have shown it to be extremely low. In addition, this inductive effect is applied only for a very short time, when the pacing pulse is being generated. Taking this effect into account would unnecessarily complicate the model.

If this circuit is supplied by a square pulse with a constant voltage $\mathrm{V}$, the current I through the circuit is dependent on time and satisfies the following first order differential equation: 


$$
\mathrm{rC} \frac{\mathrm{dI}(\mathrm{t})}{\mathrm{dt}}+\mathrm{I}(\mathrm{t})\left(1+\frac{\mathrm{r}}{\mathrm{R}}\right)=\mathrm{C} \frac{\mathrm{dV}(\mathrm{t})}{\mathrm{dt}}+\frac{\mathrm{V}}{\mathrm{R}}
$$

the solution of which is:

$$
I(t)=V\left[\frac{1}{R+r}+\left(\frac{1}{r}-\frac{1}{R+r}\right) e^{-t\left(\frac{1}{r C}+\frac{1}{R C}\right)}\right]
$$

which can be written:

$$
\mathrm{I}(\mathrm{t})=\mathrm{V}\left(\mathrm{a} \cdot \mathrm{e}^{\mathrm{bt}}+\mathrm{c}\right)
$$

where $\mathrm{a}, \mathrm{b}$ and $\mathrm{c}$ are coefficients that depend linearly on $\mathrm{R}, \mathrm{r}$ and $\mathrm{C}$. These three coefficients, $\mathrm{a}, \mathrm{b}$ and $\mathrm{c}$, form three linear equations with three unknowns, $\mathrm{R}, \mathrm{r}$ and $\mathrm{C}$. Knowing the values of $a, b$ and $c$, it is very easy to calculate the values of $\mathrm{R}, \mathrm{r}$ and $\mathrm{C}$.

The problem is to evaluate coefficients $a, b$ and $c$ knowing the current $I(t)$. This evaluation is made by nonlinear regression fit. The current is evaluated by measuring the voltage with respect to time $\mathrm{V}(\mathrm{t})$ across a measurement resistor $r_{\text {measurement }}$ connected in series in the circuit. The ratio $\mathrm{V}(\mathrm{t}) / \mathrm{r}_{\text {measurement }}$ gives the current $\mathrm{I}(\mathrm{t})$.

\section{Equipment and methods}

The constant voltage square pulse of $2 \mathrm{~ms}$ duration with a known, adjustable amplitude, is generated using the analog output of a National Instruments card. This card is installed in a PC that measures and digitizes the $\mathrm{I}(\mathrm{t})$ flowing through the circuit (1 MHz sampling rate).

Resistor $r_{\text {measurement }}$ connected in series in the circuit has a value of $100 \Omega$.

All the calculations and programming of the analog output were written in the National Instruments $G$ LabVIEW programming language. The programming creates a virtual instrument used to:

- display the constant voltage pulse,

- display the current flowing through the circuit,

- record the current in an electronic file,

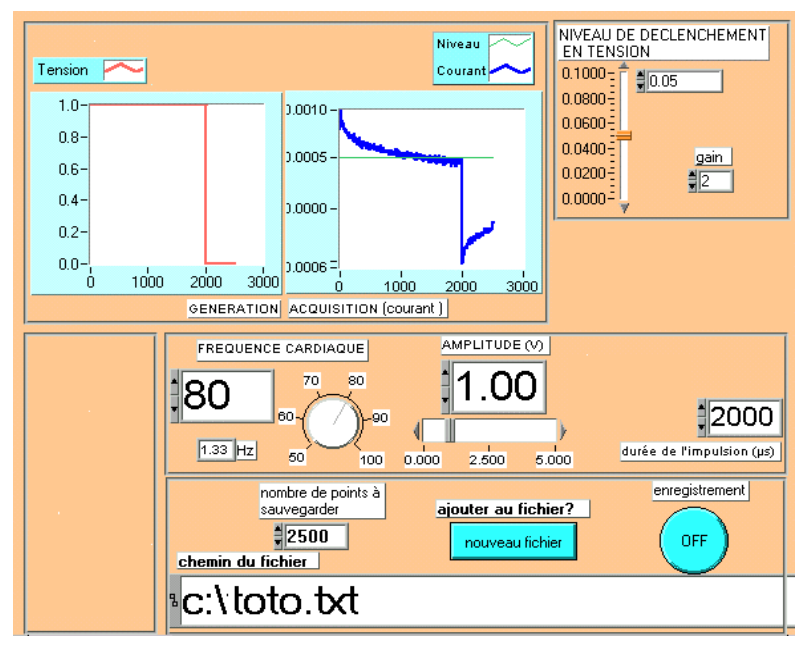

- adjust the control settings (pulse voltage, pulse duration, pacing rate, acquisition trigger level, filename to be saved, etc.).

A second virtual instrument that displays the saved file, is used to curve-fit the current $I(t)$ using a nonlinear regression algorithm (Levenberg-Marquardt algorithm) and gives the values of circuit components $\mathrm{R}, \mathrm{r}$ and $\mathrm{C}$. These values can be saved in a file for future use.

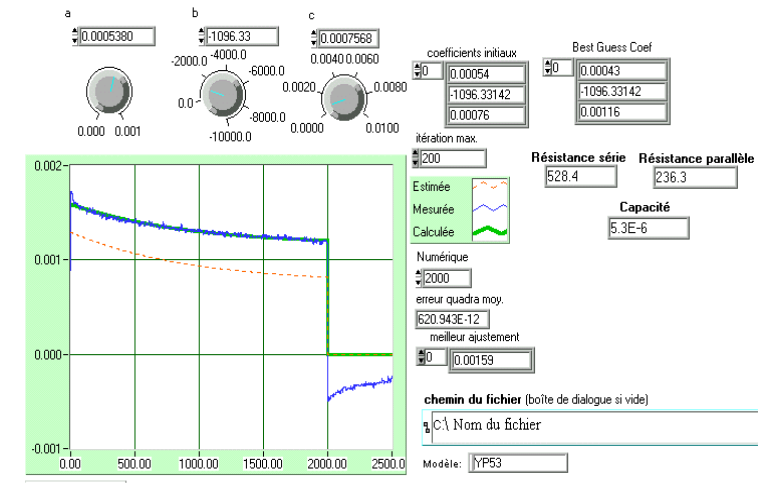

\section{Examples of results}

a) Comparison of the impedance components for three measurement conditions (discounting the wire resistance) is given in the figure below:

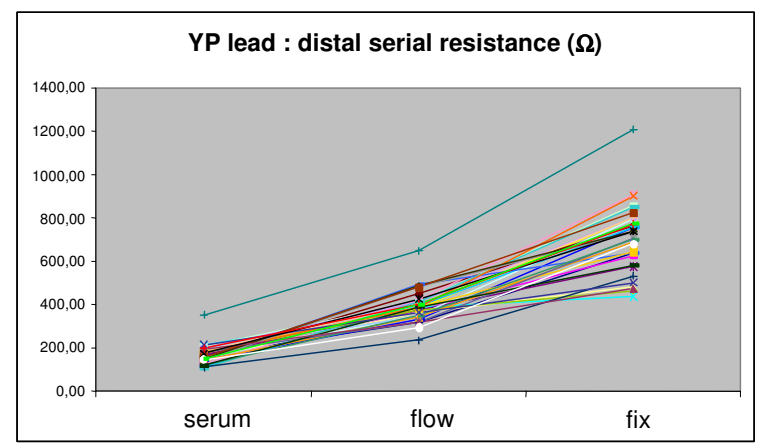

Measurements were made under the three following conditions for several types of leads:

- in a metal cup containing a physiological saline solution (serum),

- with the lead floating in the right atrium (float),

- with the lead in contact with the right ventricular endocardium after checking for a correct pacing threshold (fix).

A comparison made on several dozen leads shows an increase of around $280 \Omega$ between the saline solution and the right atrium and $200 \Omega$ between the right atrium and the right ventricle for the distal electrode. It can therefore be estimated that the blood and thorax represent a series 
resistance of $280 \Omega$ and that the endocardium adds another $200 \Omega$. These observations show that it is illusory to attempt to decrease the wire resistance, because the gain in doing so does not exceed a few tens of ohms.

b) Comparison between the capacitance of the distal electrode in contact with the endocardium for several leads:

The distal capacitances are shown in the figure below for the YP (Biotronik), ActFix (Vitatron), Fine Line II (Guidant) and Elox (Biotronik) leads. The differences between leads are significant except between YP and ActFix. The macroscopic surface areas of the above electrodes are 7, 4.2, 5 and $9.2 \mathrm{~mm} 3$. respectively. It can be seen that there is no relation between surface area and capacitance except for the Elox probe, which has both the highest capacitance and largest surface area. This lack of correlation between macroscopic surface area and capacitance is due to the surface treatments of the electrodes designed to increase the macroscopic surface area in contact with the electrolytes and tissues.

The figure below compares the capacitances of the four leads:
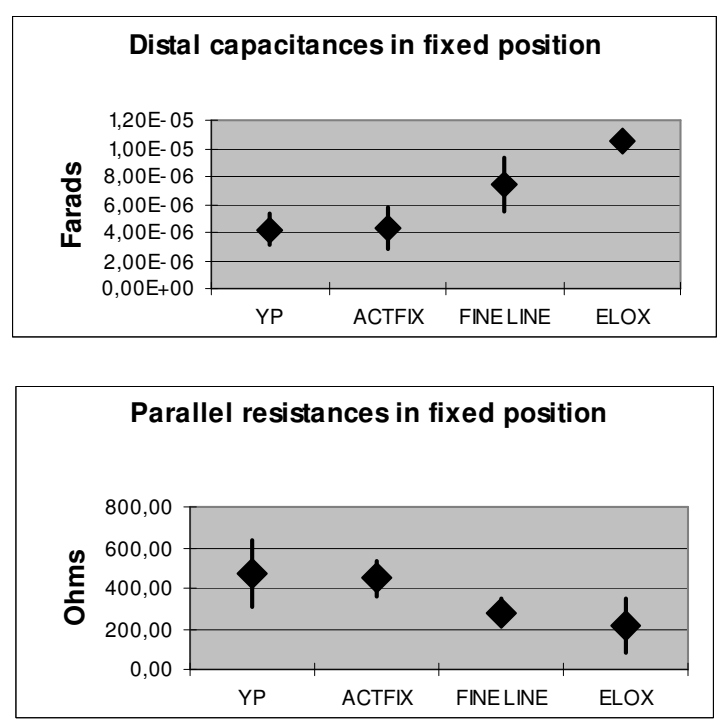

c) Comparison of different sorts of parallel resistances.

The differences between leads resistances are significant except between YP and ActFix (as was already the case for the capacitances). The parallel resistance is observed to be inversely proportional to the capacitance, in approximately the same proportions (i.e. when $\mathrm{C}$ doubles, $\mathrm{R}$ is divided by 2 and vice versa) such that product $\mathrm{RC}$ remains roughly constant. Product $\mathrm{RC}$ (time constant) participates in discharge of the capacitance through the parallel resistance and accounts for the electrode polarization properties.

\section{Discussion and advantages}

This method for measuring the heart-electrode interface allows analysis of the three model components.

These data are used to make comparisons between electrodes. It will be possible to establish relations between manufacturing techniques and certain electrical characteristics. The method can also be used for inprocess inspections. In addition, it allows analysis of the role of the thorax, blood and endocardium for certain characteristics.

A fundamental consequence of this analysis is to propose a universal definition of electrode polarization. In effect, knowing the model components, it is possible to calculate the polarization voltage at any time and from any applied current or voltage signal.

\section{References}

[1] Gressard A. Electricité et signaux. 12e Cours de perfectionnement en stimulation cardiaque. Stimucoeur 1995; 23, $\mathrm{N}^{\circ} 3.235-243$.

[2] Gressard A, Busillet $\mathrm{H}, \ldots$. Milon $\mathrm{H}$. Changes in the impedance of the lead-heart electrode interface during the stimulation pulse. Abstract Cardiostim 1998 172-p2 Europace.

[3] Gressard A, Andrivet P, Milon H. Serial resistor of pacing electrode-endocardial interface: evaluation of determinants. Abstract Cardiostim 2002. Europace 212.

[4] Irnich W. Paradigm Shift in lead design. Pacing Clin Electrophysiol. 1999 Sep;22(9):1321-32.

[5] Roelke M, Bernstein AD, Parsonnet V. Serial lead impedance measurements confirm fixation of helical screw electrodes during pacemaker implantation. Pacing Clin Electrophysiol. 2000 Apr;23(4 Pt 1):488-92.

[6] Schaldach M. The stimulating electrode. In: Electrotherapy of the heart. Berlin: Springer Verlag, 1992:145-68.

[7] Lau C. Intraoperative study of polarization and evoked response signals in different endocardial electrode designs. Pacing Clin Electrophysiol. 2001 Jul;24(7):1055-60.

Address for correspondence

Alain Gressard

Hôpital de la Croix-Rousse, Service de Cardiologie

103 Boulevard de le Croix-rousse

69317 Lyon cedex 04 France

E-mail address: alain.gressard@ @hu-lyon.fr 